\title{
Can consumer wearable activity tracker- based interventions improve physical activity and cardiometabolic health in patients with chronic diseases? A systematic review and meta-analysis of randomised controlled trials
}

Wouter M. A. Franssen ${ }^{1,2^{*}}$, Gregor H. L. M. Franssen ${ }^{3}$, Jan Spaas², Francesca Solmi ${ }^{4}$ and Bert O. Eijnde 2,5

\begin{abstract}
Background: To date, it is unclear if consumer wearable activity trackers (CWATs), with or without behaviour multicomponent strategies, effectively improve adherence to physical activity and health outcomes under free living conditions in populations with chronic diseases. Therefore, we systematically evaluated the efficacy of CWAT-based interventions to promote physical activity levels and cardiometabolic health in populations with chronic diseases.

Methods: Randomised controlled trials were collected from five bibliographic databases (PubMed, Embase, Web of Science, The Cochrane Central Register of Controlled Trials and CINAHL). Studies were eligible for inclusion if they evaluated a CWAT-based counselling intervention versus control intervention among patients with chronic respiratory diseases, type 2 diabetes mellitus, cardiovascular diseases, overweight/obesity, cognitive disorders, or sedentary older adults. Data were pooled using a random-effects model.

Results: After deduplication 8147 were identified of which 35 studies met inclusion criteria (chronic respiratory diseases: 7, type 2 diabetes mellitus: 12, cardiovascular diseases: 6, overweight/obesity: 3, cognitive disorders: 1, sedentary older adults: 6). Compared to control groups, CWAT-based interventions significantly increased physical activity by 2123 steps per day (95\% confidence interval [Cl], [1605-2641]; $p<0.001$ ). In addition, CWAT-based interventions in these populations significantly decreased systolic blood pressure $(-3.79 \mathrm{~mm} \mathrm{Hg} ; 95 \% \mathrm{Cl}$ : [- 4.53, 3.04] $\mathrm{mm} \mathrm{Hg} ; p<0.001)$, waist circumference $(-0.99 \mathrm{~cm} ; 95 \% \mathrm{Cl}:[-1.48,-0.50] \mathrm{cm} ; \mathrm{p}<0.001)$ and low-density lipoprotein cholesterol concentration $(-5.70 \mathrm{mg} / \mathrm{dl} ; 95 \% \mathrm{Cl}$ : $[-9.24,-2.15] \mathrm{mg} / \mathrm{dl} ; p=0.002)$.

(Continued on next page)
\end{abstract}

\footnotetext{
* Correspondence: Wouter.franssen@uhasselt.be

${ }^{1}$ REVAL - Rehabilitation Research Center, Faculty of Rehabilitation Sciences, Hasselt University, Hasselt, Belgium

${ }^{2}$ BIOMED - Biomedical Research Institute, Faculty of Medicine and Life Sciences, Hasselt University, Hasselt, Belgium

Full list of author information is available at the end of the article
}

(c) The Author(s). 2020 Open Access This article is licensed under a Creative Commons Attribution 4.0 International License, which permits use, sharing, adaptation, distribution and reproduction in any medium or format, as long as you give appropriate credit to the original author(s) and the source, provide a link to the Creative Commons licence, and indicate if changes were made. The images or other third party material in this article are included in the article's Creative Commons licence, unless indicated otherwise in a credit line to the material. If material is not included in the article's Creative Commons licence and your intended use is not permitted by statutory regulation or exceeds the permitted use, you will need to obtain permission directly from the copyright holder. To view a copy of this licence, visit http://creativecommons.org/licenses/by/4.0/ The Creative Commons Public Domain Dedication waiver (http://creativecommons.org/publicdomain/zero/1.0/) applies to the data made available in this article, unless otherwise stated in a credit line to the data. 
(Continued from previous page)

Conclusion: CWAT-based interventions increase physical activity and have beneficial effects on important healthrelated outcomes such as systolic blood pressure, waist circumference and LDL cholesterol concentration in patients with chronic diseases.

Keywords: Activity tracker, Physical activity, Cardiometabolic health, Chronic disease

\section{Summary box}

\section{What is already known?}

- Physical inactivity is one of the major contributing factors for the development of chronic diseases and highly correlated with mortality and hospitalization

- Consumer wearable activity trackers have predominantly been applied in the sports community for self-monitoring sport/training performance-related parameters

\section{What are the new findings?}

- Consumer wearable activity tracker-based interventions increase physical activity

- The increased number of steps could result in an improvement of the general health and a reduction of all-cause mortality in people with chronic diseases

\section{Introduction}

Chronic diseases such as chronic respiratory diseases, type 2 diabetes mellitus (T2DM), cardiovascular diseases (CVD) and cognitive disorders are an important public health concern worldwide [1]. In fact, recent data indicate that the prevalence of chronic diseases has increased considerably to 40 million global deaths in 2015. As a result, chronic diseases now are the leading cause of mortality worldwide and as such constitute one of the most important challenges for the twenty-first century $[2,3]$.

Physical inactivity is one of the major contributing factors for the development of chronic diseases and is highly correlated with mortality and hospitalization [4, 5]. On the other hand, it is well known that increased physical activity has significant health benefits and is associated with the prevention and delayed onset of many chronic diseases [4, 6]. Given the important role of physical activity in the prevention and management of chronic diseases, it is crucial to promote physical activity. Hence, to date, a multitude of physical activity recommendations and many supervised training interventions and rehabilitation programs are available to encourage physical activity in the global population $[7,8]$. Nevertheless, a recent report from the World Health Organization (WHO) indicates that $23 \%$ of the adult and $80 \%$ of the adolescent population is still physically inactive [9]. Here, very poor long-term compliance to adequate physical activity and a healthy lifestyle appears to be one of the main factors explaining this discrepancy.
Consequently, any strategy that improves long-term adherence to adequate daily physical activity and a healthy lifestyle, especially in a population with chronic diseases, is worthwhile investigating. The use of structured behaviour change interventions (e.g. telephone counselling, group sessions, provision of written information materials or individual education sessions) is reported to be effective in increasing physical activity, subsequently leading to a reduced progression of chronic diseases [10-13]. In addition, although Bravata et al. showed that goal setting may be a key motivational factor for increasing physical activity, this conclusion was based on observational studies and healthy individuals [14]. However, these strategies are often resource-intensive and timeconsuming, factors that limit long-term adherence and usually not feasible in routine clinical care [15]. In this respect and following the recent use of pedometer and accelerometer-based remote monitoring of physical activity in patients with chronic diseases, consumer wearable activity trackers (CWATs) may be an alternative strategy to increase physical activity levels. CWATs are electronic devices used for monitoring and recording daily physical activity, although nowadays the term is also used for wearable fitness gadgets. These CWATs are consisted of pedometers (e.g. Omron and Yamax), which provide direct feedback on the level of physical activity in terms of number of steps per day, and activity trackers (Polar, Fitbit, Garmin and Apple Watch) which often include goal setting and can monitor physical activity and fitness related metrics including the amount and intensity of physical activity, sedentary behaviour and heart rate. Initially, CWATs have predominantly been applied in the sports community for selfmonitoring sport/training performance-related parameters [16-18]. To date, they are widely used to quantify physical activity and monitor fitness. Possibly, the selfmanagement, motivational and goal setting properties of these commercially available devices may also help patients with chronic diseases to better adhere to longterm physical activity under free-living conditions in a home-based setting. Surprisingly and despite the widespread use of these wearables, their feasibility and efficacy on physical activity (compliance) and cardiometabolic health including anthropometric measurements, systemic blood pressure, lipid profile and glycemic index, especially in patients with chronic diseases, is not fully 
clear. Although recent reviews have shown that CWATs have potential to increase physical activity, they included only one type of chronic disease $[19,20]$ or solely pedometers $[14,21]$. In addition, conclusions are not based on randomised controlled trials $[14,22]$ and most of reviews did not (or partly) report a cardiometabolic risk profile $[15,19,20,23]$.

Therefore, this study aims to systematically evaluate the efficacy of CWAT-based interventions, as CWATs being either the primary components of an intervention or as part of a multi-component intervention, to promote physical activity levels and cardiometabolic health in populations with chronic diseases including chronic respiratory diseases, T2DM, CVD, overweight/obesity, cognitive disorders or sedentary older adults. A better understanding whether CWATs improve adherence to physical activity and hereby affect cardiometabolic health outcomes may help to increase the efficacy and quality of health care in populations with chronic diseases.

\section{Methods}

This systematic review, including an explorative metaanalysis, was registered in the PROSPERO international prospective register of systematic reviews (registration number: CRD42019124126) and was performed in accordance with The Preferred Reporting Items for Systematic reviews and Meta-Analysis (PRISMA) statement [24, 25].

\section{Data sources and search strategies}

Studies were collected (from inception until March 2019) using computer-based searches in the PubMed, Embase, Web of Science, The Cochrane Central Register of Controlled Trials (CENTRAL) and CINAHL electronic databases. Database specific search strategies were developed with the guidance of a professional clinical librarian. The database searches were performed using four main concepts: chronic diseases or sedentary older adults, CWATs, behaviour change and cardiometabolic health measures. For each main concept relevant related terms and keywords were included in the sensitive search (details presented in Appendix I). The systematic search was limited to the English, German and Dutch language.

\section{Eligibility criteria}

Inclusion criteria to select studies were: 1) Study population: adult (aged 18 or older) patients with main chronic diseases including chronic respiratory diseases, T2DM, CVD, overweight/obesity and cognitive disorders, or sedentary older adults ( $>55$ years; high risk population); 2 ) Types of studies: peer-reviewed randomised controlled trials regarding a CWAT-based behaviour change intervention compared to a control intervention or usual care comparison group. The behaviour change intervention could be a CWAT-only intervention or a multicomponent intervention consisting of a CWAT in combination with lifestyle data platforms, applications to change lifestyle behaviour or coaching sessions. In addition, physical activity should be measured objectively; 3) Primary outcome: physical activity expressed in number of steps per day and 4) Secondary outcomes: cardiometabolic health outcomes including physical fitness, exercise capacity, anthropometric measures (body weight, body mass index (BMI), waist circumference and percentage fat mass), systolic and diastolic blood pressure (BP), resting heart rate, lipid profile (blood total cholesterol, high-density lipoprotein (HDL) cholesterol, low-density lipoprotein (LDL) cholesterol and triglyceride concentrations), blood glycated haemoglobin concentration (HbA1c) and the fasting glucose and insulin concentration. Studies that included dietary interventions or had an intervention duration of less than 6 weeks were excluded.

\section{Study selection}

Studies were independently screened in three different steps by two authors (W.M.A.F. and J.S.). Firstly, duplicates were removed using the de-duplication method from Bramer et al. [26] and a first selection was performed based on titles and abstracts to identify relevant studies. Then, articles were screened and systematically excluded when they did not meet the pre-specified inclusion criteria. In addition, reviews, editorials, congress abstracts and validation studies were also excluded. Disagreements between authors were resolved by consensus with a third reviewer (B.O.E).

\section{Data extraction}

Data were independently extracted by two of the reviewers (W.M.A.F. and J.S.). Data extraction was performed with the aid of a predesigned data collection form, adapted from the extraction form of the Cochrane Collaboration (Appendix II). For each study, the reviewers extracted information with respect to study characteristics (type of study, population description, focused disease or condition, types of outcome measures); study participants (sample size, demographics); methods (study aim, study design, intervention duration, type and frequency, CWAT type, blinding, amount of intervention groups, number of included participants, dropouts and the number of individuals that were randomised and analyzed); outcome variables (outcome definition, unit of measurement, time points measured and reported, statistical method used). If a study consisted of two or more study arms of which one of the intervention arms did not meet the inclusion criteria, data were only extracted from the study arms that met the inclusion criteria. 
Continuous data including, means, standard deviations and the sample size numbers were extracted. When mean differences were not available, authors of the included studies were contacted to request additional data. Without availability of standard deviations, measures of variance were estimated from the standard error of a mean, confidence intervals or $p$-values according to the Cochrane Handbook for Systematic Reviews of Interventions the Cochrane Collaboration (Version 5.2, chapter 7) [27]. In addition, when data were presented as median and interquartile range, the mean and standard deviation were estimated using the formula from Hozo et al. [28]. Blood parameters were converted to the same unit (from $\mathrm{mmol} / \mathrm{l}$ to $\mathrm{mg} / \mathrm{dl}$ ), including triglycerides (divide by 0.0112), total cholesterol, HDL cholesterol, LDL cholesterol (divide by 0.02586 ) and glucose (divide by 0.05551 ) concentration [29].

\section{Study quality assessment}

The risk of bias of the included studies was assessed by one reviewer (W.M.A.F.) as recommended by Higgins et al. (Cochrane 'Risk of Bias' assessment tool, the Cochrane Collaboration). Here, the following methodological criteria were assessed: sequence generation, allocation concealment, blinding of participants, personnel and outcome assessors, incomplete outcome data, selective outcome reporting and other potential threats to validity [27]. Each of these criteria were judged and classified as 'low risk', 'high risk' or 'unclear risk' of bias.

\section{Statistical analysis}

Statistical analyses were performed using $\mathrm{R}$ version 3.6.0 (The $\mathrm{R}$ foundation for Statistical Computing, Wien, Austria) [30]. The mean differences (post-intervention parameter - pre-intervention parameter) with $95 \%$ confidence intervals were calculated and pooled effect estimates were obtained using a random-effects model due to the large heterogeneity among the studies (different populations, age, intervention type). Some studies used multiple intervention groups, or the same intervention and control groups were measured at multiple time intervals. Therefore, an extension of the standard metaanalysis models was applied on these data, in order to take correlations of effect estimates coming from the same study into account. The influence of population (age, sex proportion [\% male] and populations with various chronic diseases) and study characteristics (intervention type [only CWAT use, or a CWAT in combination with behaviour change strategies including goal setting, telephone support and/or individual or group counselling] and intervention duration) on the intervention effect was evaluated using a mixed effects meta-regression analysis. Here, the effects of these covariates were studied as fixed effects in the model. Tests for overall effect as well as pairwise comparisons among populations were performed. For the latter, adjustments for all other effects and corrections for multiple comparisons were used. Studies with missing values regarding the covariates were not included into the analysis. The beta estimate refers to the change in number of steps per day for a unit change in the characteristics. Sensitivity analyses were performed to assess heterogeneity of the studies and to evaluate the robustness of the results. Each study was individually removed to evaluate the effect of that study on the summary estimates. Publication bias was assessed using funnel plots and the Egger test (Appendix III). The effect of heterogeneity of each summary effect size was quantified using a chi-squared test and the $\mathrm{I}^{2}$ statistic, in which the boundary limits 25, 50, and $75 \%$ were designated as a low, moderate, and high heterogeneity value [31].

\section{Results}

The search strategy identified 13,688 potentially relevant studies of which 8147 after deduplication (Fig. 1). Thirty-five full-text articles fulfilled the inclusion criteria and were included in qualitative $(n=35)$ and quantitative $(n=33)$ synthesis (Table 1.). Studies included were published over a 14-year period from 2004 to 2018. All studies were written in English and performed in 13 different countries predominantly originating from the United States $(n=10)$. The included studies consisted of populations with various chronic diseases including chronic respiratory diseases $(n=7)$ [32-38], impaired glucose tolerance or T2DM $(n=12)$ [39-50], CVD $(n=$ 6) [51-56], overweight/obesity $(n=3)$ [57-59], cognitive disorders $(n=1)[60]$ and sedentary older adults $(n=6)$ [61-65].

\section{Risk of bias}

Among the 35 included studies, several increased risks of bias were assessed. Fifteen studies met five $(n=5)$ [37, $42,57,59,65]$ and four $(n=10)[18,33,38,41,49,53$, $55,58,64,66]$ of the six risk of bias criteria. Eighteen studies met three criteria [32, 34-36, 39, 40, 43-45, 47, $50-52,56,60-63]$ and two studies met only two criteria $[48,54]$. Although all studies reported appropriate random sequence allocation, insufficient information regarding the procedures used to conceal the allocation of the different trial $\operatorname{arm}(\mathrm{s})$ was provided in 21 of the 35 studies (Fig. 2). The largest risk of bias was with regard to the performance bias (lack of blinding of participants and personnel) and detection bias (minimal blinding of those assessing outcomes). All included studies were unable to blind the participants and the study personnel/ physicians $(n=18)$ or to adequately report blinding $(n=$ 17). Only eight studies blinded the outcome assessors, whereas the majority of the studies did not adequately 
Table 1 Characteristics of included studies

\begin{tabular}{ll}
\hline Study & Population \\
& \\
\hline Altenburg & COPD $\left(62 \pm 1^{\text {a }}\right.$ years; \\
$\mathbf{2 0 1 5}$ & sex $\mathrm{m} / \mathrm{f}:$ 102/53)
\end{tabular}

2015 sex m/f: 102/53)

Araiza

2006

T2DM (50 \pm 10 years)

\section{Intervention}

duration

(wk)

64

6

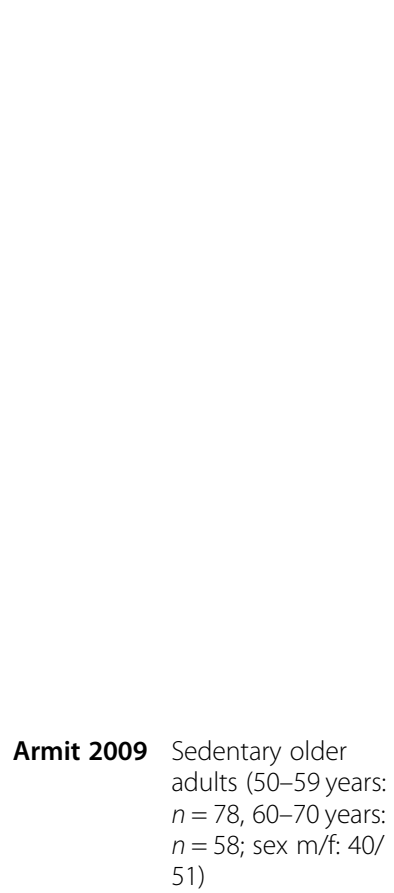

51)

Baker 2008 Overweight/Obesity $(49.3 \pm 8.8$ years; sex m/f: 63/16)

12

$0 \quad 46$

$12 \quad 20 \quad 39$

\begin{tabular}{|c|c|}
\hline $\begin{array}{l}\text { Dropout } \\
\text { rate }(\%)\end{array}$ & $\begin{array}{l}\text { No. of } \\
\text { participants }\end{array}$ \\
\hline 30 & 78 \\
\hline
\end{tabular}

77

setting, motivational

(five individual

30 min counselling sessions) and

pedometer use for feedback and

motivation

77

C: Usual care

I: Pedometer use and instructed to walk 10.000 steps/day on 5 or more days of the week

\section{CWAT type Outcome}

parameter

Physical activity:

Digiwalker - Steps/day

SW-200

Digiwalker - Steps/day

SW-701 Cardiometabolic

risk:

- Body mass

index

- Waist

circumference

- Body fat

percentage

- Systolic blood

pressure

- Diastolic blood

pressure

- HbA1c

- Fasting glucose

- Fasting insulin

- HOMA-IR

- Triglycerides

- Total

cholesterol

- HDL

cholesterol

- LDL cholesterol

15 C: Pedometer use and instructed to maintain normal activity habits

I: Physical activity advice provided during a 30 min behaviour change counselling session (Transtheoritical model) and three follow-up telephone calls, pedometer use, goal setting and motivational interviewing techniques

C: Usual care (3-5 min of brief verbal physical activity advice and written information)
I: Pedometer-based walking program with consultations based on the Transtheoretical Model of exercise behaviour change, goal setting (3000 steps/ day above baseline) mron HJ- Physical activity: 109E Step-O- - Steps/day Meter Cardiometabolic risk:

- Body weight

- Body mass

index

- Waist

circumference

- Hip

circumference

- Waist-to-hip

ratio

- Body fat

percentage

- Systolic blood

pressure

- Diastolic blood 
Table 1 Characteristics of included studies (Continued)

\begin{tabular}{|c|c|c|c|c|c|c|c|}
\hline Study & Population & $\begin{array}{l}\text { Intervention } \\
\text { duration } \\
\text { (wk) }\end{array}$ & $\begin{array}{l}\text { Dropout } \\
\text { rate (\%) }\end{array}$ & $\begin{array}{l}\text { No. of } \\
\text { participants }\end{array}$ & Intervention & CWAT type & $\begin{array}{l}\text { Outcome } \\
\text { parameter }\end{array}$ \\
\hline & & & & & & & $\begin{array}{l}\text { pressure } \\
\text { - Resting heart } \\
\text { rate } \\
\text { - Total } \\
\text { cholesterol } \\
\text { - HDL } \\
\text { cholesterol } \\
\text { - LDL cholesterol }\end{array}$ \\
\hline & & & & 40 & C: Maintain usual activity levels & & \\
\hline $\begin{array}{l}\text { Bjorgaas } \\
2008\end{array}$ & $\begin{array}{l}\text { T2DM }(58.9 \pm 10.5 \\
\text { years; sex m/f: } 31 / 17)\end{array}$ & 26 & 31 & 23 & $\begin{array}{l}\text { I: Pedometer use, goal setting, physical } \\
\text { activity calendar, encouraged to } \\
\text { increase targeted number of steps }\end{array}$ & $\begin{array}{l}\text { Yamax } \\
\text { Digiwalker } \\
\text { ML AW-320 }\end{array}$ & $\begin{array}{l}\text { Cardiometabolic } \\
\text { risk: } \\
\text { - Body weight } \\
\text { - Systolic blood } \\
\text { pressure } \\
\text { - Diastolic blood } \\
\text { pressure } \\
\text { - HbA1c } \\
\text { - Fasting glucose } \\
\text { - Triglycerides } \\
\text { - Total } \\
\text { cholesterol } \\
\text { - HDL } \\
\text { cholesterol } \\
\text { - Peak oxygen } \\
\text { uptake }\end{array}$ \\
\hline
\end{tabular}

Bond 2014 Overweight/Obesity $(46.0 \pm 8.9$ years; sex m/f: 10/65) spent walking

I: Individual face-to-face counseling sessions (6 sessions lasted $30-45 \mathrm{~min}$ ) based on theoretical constructs from the Transtheoretical Model, Theory of Planned Behaviour, Social Cognitive Theory, and Self-Determination Theory; goal setting, pedometer use, problem solving, action planning, review of progress

C:Standard preoperative care. Surgeons and members of the surgical team advised

participants to adopt an active lifestyle and engage in walking exercise and other similar activities

I: Pedometer use, step calendar, four telephone calls, behavioural counselling, goal setting, generic physical activity information brochures

C: Generic physical activity information brochures

I: Individual standardized educational session, Pedometer use, step based physical activity prescription, goal setting (steps taken during the previous week plus 1000 steps)

17 C: Individual standardized educational session

$\begin{array}{ll}\text { I: Behaviour change counselling } & \text { Yamax } \\ \text { session (Social cognitive theory), } & \text { Digiwalker } \\ \text { pedometer use, goal setting, select } & \text { SW-200 }\end{array}$

Physical activity: - Steps/day
Yamax
Digiwalker $700 \mathrm{~B}$

Cardiometabolic risk:

- Body mass

index

- Waist

circumference

Yamax

Digiwalker SW-200

Physical activity: - Steps/day 
Table 1 Characteristics of included studies (Continued)

\begin{tabular}{|c|c|c|c|c|c|c|}
\hline Study & Population & $\begin{array}{l}\text { Intervention } \\
\text { duration } \\
(w k)\end{array}$ & $\begin{array}{l}\text { Dropout } \\
\text { rate (\%) }\end{array}$ & $\begin{array}{l}\text { No. of } \\
\text { participants }\end{array}$ & Intervention & $\begin{array}{l}\text { Outcome } \\
\text { parameter }\end{array}$ \\
\hline
\end{tabular}

counts, review pedometer usage and discuss procedures for keeping a step calendar and monthly group sessions

$\begin{array}{ll}\text { De Blok } & \text { COPD }(64.1 \pm 11.1 \\ 2006 & \text { years } \text { sex m/f: } 9 / 12)\end{array}$

10 years; sex m/f: 9/12)

De Greef

2010 $n=6,55-75$ years: $n=35$; sex m/f: 28/ 13)

\section{De Greef \\ 2011 (1)}

T2DM $(67.4 \pm 9.3$ years; sex m/f: 47/20)

C: Instructed to continue usual activity habits

I: Behaviour change counselling programme and pedometer use for feedback and motivation

C: Usual care (regular pulmonary rehabilitation programme)

I: cognitive-behavioural group programme (5 sessions of $90 \mathrm{~min}$ ), pedometer use
T2DM (35-54 years:

Yamax

Digiwalker

Physical activity:

SW-200
C: Usual care and one single group education on the effects of physical activity on diabetes care

11: Pedometer and diary use, goal setting, three 90 min group counselling sessions (based on the cognitive behavioural therapy, the Diabetes Prevention Program, the First Step Program and motivational interviewing) by a clinical psychologist

12: Pedometer and diary use, goal setting, three individual 15 min faceto-face consultations with a clinical psychologist

C: General care from the general practitioner

I: Face-to-face session (based on the

$\begin{array}{ll}\text { Yamax } & \text { Physical activity: } \\ \text { Digiwalker } & \text { - Steps/day } \\ \text { SW-200 } & \text { Cardiometabolic } \\ & \text { risk: } \\ & \text { - Body mass } \\ & \text { index } \\ & \text { - Waist } \\ & \text { circumference } \\ & - \text { HbA1c } \\ & \text { - Fasting glucose } \\ & - \text { Total } \\ & \text { cholesterol }\end{array}$
cognitive behavioural therapy, the Diabetes Prevention Program, the First

Yamax

Digiwalker

Physical activity: - Steps/day

SW-200
Steps/day

Digiwalker

SW-200

Cardiometabolic risk:

- Body weight

- Body mass

index

- Systolic blood

pressure

- Diastolic blood

4pressure

- HbA1c

- Total

cholesterol
Diedrich 2009
T2DM (54.2 \pm 11.6

years sex m/f: 18/35) viewing), pedometer and diary use, 24week telephone support programme

Cardiometabolic

risk: Program, Manpo-Kei book; pedometer Digiwalker use, record pedometer readings according to instructions in the book
- Body fat percentage

- Systolic blood pressure

- Diastolic blood 
Table 1 Characteristics of included studies (Continued)

\begin{tabular}{|c|c|c|c|c|c|}
\hline Study & Population & $\begin{array}{l}\text { Intervention } \\
\text { duration } \\
(w k)\end{array}$ & $\begin{array}{l}\text { Dropout } \\
\text { rate (\%) }\end{array}$ & $\begin{array}{l}\text { No. of } \\
\text { participants }\end{array}$ & Intervention \\
\hline & & & & 26 & $\begin{array}{l}\text { C: Diabetes Self-Management Educa- } \\
\text { tion Program }\end{array}$ \\
\hline \multirow[t]{2}{*}{$\begin{array}{l}\text { Duscha } \\
2018\end{array}$} & $\begin{array}{l}\text { CVD (62.3 } \pm 8.3 \text { years; } \\
\text { sex m/f: 19/6) }\end{array}$ & 12 & 22 & 16 & $\begin{array}{l}\text { I: CWAT use, exercise prescription by } \\
\text { daily step count, health coaching } \\
\text { (health related recommendations, } \\
\text { planning, motivation and sending } \\
\text { educational material via email and text } \\
\text { messages) }\end{array}$ \\
\hline & & & & 9 & C: Usual care \\
\hline Engel 2006 & $\begin{array}{l}\text { T2DM (62.4 } \pm 7.7 \\
\text { years; sex m/f: } 28 / 26)\end{array}$ & 26 & 7 & 24 & $\begin{array}{l}\text { I: Coaching (Diabetes-related } \\
\text { education, goal setting, supportive/ } \\
\text { motivational strategies, behaviour } \\
\text { change strategies and psychosocial } \\
\text { support by telephone or face-to-face } \\
\text { contact) and pedometer use }\end{array}$ \\
\hline
\end{tabular}

CWAT type Outcome

parameter

pressure

- HbA1c

Fitbit Charge Physical activity: - Steps/day

Cardiometabolic:

- Peak oxygen

uptake

$\begin{array}{ll}\text { Yamax } & \text { Cardiometabolic: } \\ \text { Digiwalker- } & \text { - Body weight } \\ 700 & \text { - Body mass } \\ & \text { index } \\ & \text { - Waist } \\ & \text { circumference } \\ & \text { - Systolic blood } \\ & \text { pressure } \\ & \text { - Diastolic blood } \\ & \text { pressure } \\ \text { - HbA1c }\end{array}$

$30 \quad$ C: Coaching (Diabetes-related education, goal setting, supportive/ motivational strategies, behaviour change strategies and psychosocial support by telephone or face-to-face contact)

$\begin{array}{lllll}\text { Hospes } & \text { COPD }(62.2 \pm 8.6 & 12 & 10 & 18 \\ \mathbf{2 0 0 9} & \text { years; sex m/f: } 21 / 14) & & & \\ & & & & 17 \\ \text { Houle 2011 } & \text { CVD }(58.5 \pm 8.5 \text { years; } & 52 & 22 & 32 \\ & \text { sex m/f: } 51 / 14)\end{array}$

I: Customized exercise counselling programme (5 sessions), goal setting and pedometer use

C: Usual care

I: Pedometer use, diary, physical activity, goal setting (target of 3000 steps per day increment in physical activity counselling (social cognitive theory) by a clinical nurse specialist

$\begin{array}{ll}\text { Digiwalker } & \text { Physical activity: } \\ \text { SW-200 } & \text { - Steps/day } \\ & \text { Cardiometabolic: } \\ & \text { - Waist } \\ & \text { circumference } \\ & \text { - Systolic blood } \\ \text { pressure } & \text { - Diastolic blood } \\ & \text { pressure } \\ \text { - Resting heart } & \text { rate } \\ \text { - Fasting glucose } & \text { - Triglycerides } \\ \text { - LDL cholesterol } \\ \text { - HDL } \\ \text { cholesterol }\end{array}$

33 C: Usual care

Kaminsky CVD (56.0 \pm 9.2 years;

8

I: Recommended to obtain a minimum of 30-40 min/day moderate-to-

NL-1000

Newvigorous physical activity on days they lifestyles did not attend cardiac rehabilitation, daily step count goals to increase by $10 \%$ of baseline step per day

C: Usual care

I: Multidisciplinary home-based physical rehabilitation program, pedometer use, goal of 8000 steps per day, staff gave verbal reinforcement
Digiwalker Physical activity: SW-200 - Steps/day
Physical activity: - Steps/day 
Table 1 Characteristics of included studies (Continued)

\begin{tabular}{|c|c|c|c|c|c|c|c|}
\hline Study & Population & $\begin{array}{l}\text { Intervention } \\
\text { duration } \\
\text { (wk) }\end{array}$ & $\begin{array}{l}\text { Dropout } \\
\text { rate (\%) }\end{array}$ & $\begin{array}{l}\text { No. of } \\
\text { participants }\end{array}$ & Intervention & CWAT type & $\begin{array}{l}\text { Outcome } \\
\text { parameter }\end{array}$ \\
\hline & & & & 15 & $\begin{array}{l}\text { C: Multidisciplinary home-based phys- } \\
\text { ical rehabilitation program }\end{array}$ & & \\
\hline \multirow[t]{3}{*}{$\begin{array}{l}\text { Kempf } \\
2018\end{array}$} & $\begin{array}{l}\text { Overweight/obese } \\
(45.4 \pm 10.0 \text { years; sex } \\
\text { m/f: } 81 / 99)\end{array}$ & 12 & 16 & 58 & $\begin{array}{l}\text { 11: Pedometer use, weekly care calls } \\
\text { from trained coaches (information } \\
\text { about overweight or obesity-related } \\
\text { diseases like type } 2 \text { diabetes, healthy } \\
\text { diet, physical activity, and coping strat- } \\
\text { egies for lifestyle changes), participants } \\
\text { were motivated to achieve individual } \\
\text { goals using mental motivation } \\
\text { program }\end{array}$ & $\begin{array}{l}\text { SmartLAB } \\
\text { walk P+ }\end{array}$ & $\begin{array}{l}\text { Cardiometabolic } \\
\text { risk: } \\
\text { - Body weight } \\
\text { - Body mass } \\
\text { index } \\
\text { - Waist } \\
\text { circumference } \\
\text { - Systolic blood } \\
\text { pressure } \\
\text { - Diastolic blood } \\
\text { pressure } \\
\text { - HbA1c } \\
\text { - Triglycerides } \\
\text { - Total } \\
\text { cholesterol } \\
\text { - HDL } \\
\text { cholesterol } \\
\text { - LDL cholesterol }\end{array}$ \\
\hline & & & & 61 & 12: Pedometer use & & \\
\hline & & & & 61 & C: Routine care & & \\
\hline \multirow[t]{2}{*}{$\begin{array}{l}\text { Kooiman } \\
2018\end{array}$} & $\begin{array}{l}\text { T2DM }(56.4 \pm 11.3 \\
\text { years; sex m/f: } 38 / 34)\end{array}$ & 13 & 8 & 40 & $\begin{array}{l}\text { I: Usual care, activity tracker and access } \\
\text { to online self-tracking (eHealth) } \\
\text { program }\end{array}$ & Fitbit Zip & $\begin{array}{l}\text { Cardiometabolic } \\
\text { risk: } \\
\text { - Body weight } \\
\text { - Body mass } \\
\text { index } \\
\text { - Waist } \\
\text { circumference } \\
\text { - Hip } \\
\text { circumference } \\
\text { - Waist-to-hip } \\
\text { ratio } \\
\text { - HbA1c }\end{array}$ \\
\hline & & & & 32 & C: Usual care & & \\
\hline \multirow[t]{2}{*}{$\begin{array}{l}\text { Mendoza } \\
2015\end{array}$} & $\begin{array}{l}\operatorname{COPD}(68.7 \pm 8.5 \\
\text { years; sex m/f: } 62 / 40)\end{array}$ & 12 & 5 & 52 & $\begin{array}{l}\text { I: Pedometer use, diary with step } \\
\text { count, goal setting }\end{array}$ & $\begin{array}{l}\text { Tanita PD724 } \\
\text { Triaxial } \\
\text { pedometer }\end{array}$ & $\begin{array}{l}\text { Physical activity: } \\
\text { - Steps/day }\end{array}$ \\
\hline & & & & 50 & $\begin{array}{l}\text { C: Counselling to increase physical } \\
\text { activity (to walk at least } 30 \mathrm{~min} / \text { day) }\end{array}$ & & \\
\hline \multirow[t]{2}{*}{ Nolan 2017} & $\begin{array}{l}\text { COPD ( } 68 \pm 9 \text { years; } \\
\text { sex m/f: } 110 / 42)\end{array}$ & 26 & 26 & 76 & $\begin{array}{l}\text { I: Usual care (physical rehabilitation } \\
\text { programme), pedometer use, goal } \\
\text { setting and step count diary }\end{array}$ & $\begin{array}{l}\text { Yamax } \\
\text { Digiwalker } \\
\text { CW700 }\end{array}$ & $\begin{array}{l}\text { Physical activity: } \\
\text { - Steps/day }\end{array}$ \\
\hline & & & & 76 & $\begin{array}{l}\text { C: Usual care (physical rehabilitation } \\
\text { programme) }\end{array}$ & & \\
\hline \multirow[t]{3}{*}{$\begin{array}{l}\text { Rowley } \\
2017\end{array}$} & $\begin{array}{l}\text { Sedentary older } \\
\text { adults ( } 67.3 \pm 6.3 \\
\text { years; sex } \mathrm{m} / \mathrm{f}: 135 \text { / } \\
\text { 35) }\end{array}$ & 12 & 24 & 51 & $\begin{array}{l}\text { 11:Pedometer use, given the goal to } \\
\text { increase daily step count by } 10 \% \text { each } \\
\text { week, when } 10.000 \text { steps per day were } \\
\text { reached they were instructed to } \\
\text { maintain } 10.000 \text { steps per day, } \\
\text { incentives }\end{array}$ & $\begin{array}{l}\text { Omron HJ- } \\
\text { 720ITC }\end{array}$ & $\begin{array}{l}\text { Physical activity: } \\
\text { - Steps/day }\end{array}$ \\
\hline & & & & 62 & $\begin{array}{l}\text { 12: Pedometer use, interactive website } \\
\text { with key strategies to increase PA } \\
\text { systematically in older adults including } \\
\text { education and goal setting, self- } \\
\text { regulation, and frequent feedback and } \\
\text { rewarding, incentives }\end{array}$ & & \\
\hline & & & & 57 & C: Maintain usual activity levels & & \\
\hline Suboc & Sedentary older & 12 & 6 & 41 & 11: Pedometer use, goal setting (10\% & Omron HJ- & Physical activity: \\
\hline
\end{tabular}


Table 1 Characteristics of included studies (Continued)

\begin{tabular}{|c|c|c|c|c|c|c|c|}
\hline Study & Population & $\begin{array}{l}\text { Intervention } \\
\text { duration } \\
\text { (wk) }\end{array}$ & $\begin{array}{l}\text { Dropout } \\
\text { rate (\%) }\end{array}$ & $\begin{array}{l}\text { No. of } \\
\text { participants }\end{array}$ & Intervention & CWAT type & $\begin{array}{l}\text { Outcome } \\
\text { parameter }\end{array}$ \\
\hline 2014 [1] & $\begin{array}{l}\text { adults }(63.1 \pm 7.3 \\
\text { years; sex } \mathrm{m} / \mathrm{f}: 71 / 36)\end{array}$ & & & & $\begin{array}{l}\text { above baseline each week to reach } \\
10.000 \text { steps/day) }\end{array}$ & 720ITC & $\begin{array}{l}\text { - Steps/day } \\
\text { Cardiometabolic } \\
\text { risk: } \\
\text { - Body weight } \\
\text { - Body mass } \\
\text { index } \\
\text { - Waist } \\
\text { circumference } \\
\text { - Systolic blood } \\
\text { pressure } \\
\text { - Diastolic blood } \\
\text { pressure } \\
\text { - Resting heart } \\
\text { rate } \\
\text { - Fasting glucose } \\
\text { - Fasting insulin } \\
\text { - Triglycerides } \\
\text { - Total } \\
\text { cholesterol } \\
\text { - HDL } \\
\text { cholesterol } \\
\text { - LDL cholesterol }\end{array}$ \\
\hline
\end{tabular}

CVD $(59.0 \pm 8.5$ years: sex m/f: 260/64)

Tudor-

T2DM $(52.7 \pm 5.2$

Locke 2004 years; sex m/f: 26/21)

$16 \quad 22 \quad 24$

12: Pedometer use combined with interactive website intervention (frequent feedback, motivational messages, self-regulation, goal setting, education and practice in realistic behavioural change strategies, rewarding)

C: Maintain usual activity levels

11: Pedometer use, goal setting (10\% above baseline each week to reach 10.000 steps/day)

\section{Omron HJ- Physical activity: 720ITC - Steps/day \\ Cardiometabolic risk: \\ - Body weight \\ - Body mass \\ index \\ - Waist \\ circumference \\ - Systolic blood pressure \\ - Diastolic blood pressure \\ - Resting heart rate}

12: Pedometer use combined with interactive website intervention (frequent feedback, motivational messages, self-regulation, goal setting, education and practice in realistic behavioural change strategies, rewarding)

C: Maintain usual activity levels

I: Pedometer use, 3 face-to-face group formation about health behavior, selfmonitoring, goal setting, feedback, barrier identification and relapse prevention

163 C: Usual care
I: First Step behavioural modification program: Behaviour modification program based on the theoretical principles of self-efficacy and social
Digiwalker Physical activity: SW-200 - Steps/day

Cardiometabolic: - Body weight 
Table 1 Characteristics of included studies (Continued)

\begin{tabular}{|c|c|c|c|c|c|c|c|}
\hline Study & Population & $\begin{array}{l}\text { Intervention } \\
\text { duration } \\
\text { (wk) }\end{array}$ & $\begin{array}{l}\text { Dropout } \\
\text { rate (\%) }\end{array}$ & $\begin{array}{l}\text { No. of } \\
\text { participants }\end{array}$ & Intervention & CWAT type & $\begin{array}{l}\text { Outcome } \\
\text { parameter }\end{array}$ \\
\hline & & & & & $\begin{array}{l}\text { support, the common clinical practices } \\
\text { of goal setting, pedometer use and a } \\
\text { calendar for self-monitoring and } \\
\text { feedback }\end{array}$ & & $\begin{array}{l}\text { - Body mass } \\
\text { index } \\
\text { - Waist } \\
\text { circumference } \\
\text { - Hip } \\
\text { circumference } \\
\text { - Systolic blood } \\
\text { pressure } \\
\text { - Diastolic blood } \\
\text { pressure } \\
\text { - Resting heart } \\
\text { rate } \\
\text { - HbA1c } \\
\text { - Fasting glucose } \\
\text { - Fasting insulin } \\
\text { - Triglycerides } \\
\text { - Total } \\
\text { cholesterol } \\
\text { - LDL cholesterol } \\
\text { - HDL } \\
\text { cholesterol }\end{array}$ \\
\hline & & & & 23 & C: Usual care & & \\
\hline \multirow[t]{2}{*}{$\begin{array}{l}\text { Van Dyck } \\
2013\end{array}$} & $\begin{array}{l}\text { T2DM (62 } \pm 9 \text { years; } \\
\text { sex m/f: } 63 / 29)\end{array}$ & 52 & 0 & 60 & $\begin{array}{l}\text { I: Pedometer use, face-to-face session, } \\
\text { telephone support and goal setting. }\end{array}$ & $\begin{array}{l}\text { Digiwalker } \\
\text { SW-200 }\end{array}$ & $\begin{array}{l}\text { Physical activity: } \\
\text { - Steps/day } \\
\text { Cardiometabolic: } \\
\text { - Body weight } \\
\text { - Body mass } \\
\text { index } \\
\text { - Waist } \\
\text { circumference } \\
\text { - Systolic blood } \\
\text { pressure } \\
\text { - HbA1c } \\
\text { - Fasting glucose } \\
\text { - Triglycerides } \\
\text { - Total } \\
\text { cholesterol } \\
\text { - LDL cholesterol } \\
\text { - HDL } \\
\text { cholesterol }\end{array}$ \\
\hline & & & & 32 & C: Usual care & & \\
\hline \multirow[t]{2}{*}{$\begin{array}{l}\text { Vidoni } \\
2016\end{array}$} & $\begin{array}{l}\text { Cognitive } \\
\text { impairment related } \\
\text { to Alzheimer's } \\
\text { disease }(72.3 \pm 5.2 \\
\text { years; sex } \mathrm{m} / \mathrm{f}: 12 / 9)\end{array}$ & 16 & 43 & 9 & $\begin{array}{l}\text { I: Pedometer use, goal setting } \\
\text { (increase goal steps } 20 \% \text { each week } \\
\text { and maintaining in weeks } 7 \text { and 8), bi- } \\
\text { weekly phone calls to encourage phys- } \\
\text { ical activity }\end{array}$ & Fitbit Zip & $\begin{array}{l}\text { Physical activity: } \\
\text { - Steps/day }\end{array}$ \\
\hline & & & & 12 & C: Masked pedometers & & \\
\hline \multirow[t]{2}{*}{ Vogel 2017} & $\begin{array}{l}\text { CVD }(62.8 \pm 9.5 \text { years; } \\
\text { sex m/f: } 29 / 0)\end{array}$ & 12 & 0 & 13 & I: Pedometer use, goal setting & Polar loop & $\begin{array}{l}\text { Cardiometabolic: } \\
\text { - Work rate }\end{array}$ \\
\hline & & & & 16 & C: Usual care & & \\
\hline \multirow[t]{2}{*}{$\begin{array}{l}\text { Yamada } \\
2012\end{array}$} & $\begin{array}{l}\text { Sedentary older } \\
\text { adults ( } 75.7 \pm 6.8 \\
\text { years; sex m/f: } 42 / 40 \text { ) }\end{array}$ & 26 & 6 & 40 & $\begin{array}{l}\text { I: Pedometer-based behavioural } \\
\text { change program consisted of goal set- } \\
\text { ting (increase daily steps by } 10 \% \text { each } \\
\text { month), self-monitoring and feedback }\end{array}$ & $\begin{array}{l}\text { Yamax } \\
\text { Powerwalker } \\
\text { EX-510 }\end{array}$ & $\begin{array}{l}\text { Physical activity: } \\
\text { - Steps/day }\end{array}$ \\
\hline & & & & 42 & C: Maintain usual activity levels & & \\
\hline \multirow[t]{2}{*}{ Yates 2009} & $\begin{array}{l}\text { Impaired glucose } \\
\text { tolerance (65.5 } \pm 9.0 \\
\text { years; sex m/f: } 37 / 21)\end{array}$ & 52 & 18 & 29 & $\begin{array}{l}\text { I: Group-based education program } \\
\text { (PREPARE program), pedometer use, } \\
\text { goal setting (increase activity levels by } \\
\text { at least } 3000 \text { steps/day) }\end{array}$ & $\begin{array}{l}\text { Digiwalker } \\
\text { SW-200 }\end{array}$ & $\begin{array}{l}\text { Physical activity: } \\
\text { - Steps/day } \\
\text { Cardiometabolic: } \\
\text { - Fasting glucose }\end{array}$ \\
\hline & & & & 29 & C: Usual care & & \\
\hline
\end{tabular}


Table 1 Characteristics of included studies (Continued)

\begin{tabular}{|c|c|c|c|c|c|c|c|}
\hline Study & Population & $\begin{array}{l}\text { Intervention } \\
\text { duration } \\
\text { (wk) }\end{array}$ & $\begin{array}{l}\text { Dropout } \\
\text { rate (\%) }\end{array}$ & $\begin{array}{l}\text { No. of } \\
\text { participants }\end{array}$ & Intervention & CWAT type & $\begin{array}{l}\text { Outcome } \\
\text { parameter }\end{array}$ \\
\hline \multirow[t]{2}{*}{ Yates 2010} & $\begin{array}{l}\text { Impaired glucose } \\
\text { tolerance }(65.0 \pm 8.9 \\
\text { years; sex m/f: } 33 / 17)\end{array}$ & 52 & 24 & 24 & $\begin{array}{l}\text { I: Single session group-based educa- } \\
\text { tion program (PREPARE program), ped- } \\
\text { ometer use, goal setting (increase } \\
\text { activity levels by at least } 3000 \text { steps/ } \\
\text { day) }\end{array}$ & $\begin{array}{l}\text { NL-800 } \\
\text { New- } \\
\text { lifestyles }\end{array}$ & $\begin{array}{l}\text { Physical activity: } \\
\text { - Steps/day } \\
\text { Cardiometabolic: } \\
\text { - Body weight } \\
\text { - Body mass } \\
\text { index } \\
\text { - Fasting glucose } \\
\text { - Triglycerides } \\
\text { - Total } \\
\text { cholesterol } \\
\text { - HDL } \\
\text { cholesterol }\end{array}$ \\
\hline & & & & 26 & C: Usual care & & \\
\hline
\end{tabular}

report blinding of the outcome assessors $(n=22)$. Most of the studies provided complete outcome data $(n=31)$, whereas three studies had a high risk of attrition bias $[38,54,60]$ due to incomplete outcome data and only one study reported insufficient information [48].

\section{Study characteristics}

The CWAT-based interventions evaluated in the included studies varied considerably in intervention duration (mean \pm SD; $22 \pm 17$ weeks; range: 6 -64 weeks). In addition, various studies evaluated the effect of a multicomponent intervention on physical activity and cardiometabolic health, such as CWAT use with individual counselling and goal setting $(n=10)$ and CWAT use with group counselling $(n=3)[41,49,50]$. The majority of the interventions consisted of CWAT use and individual counselling $(n=22)$ of which five interventions also used additional telephone calls [48, 51, 59-61]. Control conditions varied between studies and consisted of usual care $(n=21)[32,34-36,38,41-43,46-50,52-56,58$, $59,61]$, maintaining normal physical activity levels $(n=$ 7) $[39,57,62-66]$, encouragement to increase daily physical activity $(\mathrm{n}=2)[37,40]$, generic information brochures $(n=1)$ [51], education sessions $(n=2)$ [33, 44], coaching sessions $(n=1)$ [45] and one control group wore masked CWAT devices $(n=1)$ [60]. Furthermore, the average dropout rate among the studies was $17 \%$ (range: $0-43 \%$ ) of which four studies had a 100\% participants completion rate.

\section{Population characteristics}

The included studies evaluated a total of 2858 participants (intervention: $n=1567$; control: $n=1291$ ) of which 529 participants with chronic respiratory diseases (492 with chronic obstructive pulmonary disease; COPD), 704 participants with T2DM, 571 participants with CVD, 334 participants with overweight/obesity, 21 participants with Alzheimer's disease and 699 sedentary older adults.
Their mean age was $61.3 \pm 7.3$ years (range: $45.4-75.7$ years) whereas two studies did not provide information of the average population age [41, 61]. Overall, $60 \%$ (range: $13-89 \%$ ) of the participants were male and one study included only male participants. Twenty-five studies reported daily step counts and most of the participants in the intervention ( $5105 \pm 2591$ steps/day; range: 2031-9003 steps/day) and control (5149 \pm 2751 steps/ day; range: 2334-7539 steps/day) groups were relatively physically inactive at baseline.

\section{Physical activity}

Thirty-three of the studies included data on mean differences of physical activity, expressed as steps per day, and were included in the meta-analyses. Overall, participants with chronic diseases assigned to an intervention group significantly increased their physical activity (step count) by 2123 steps per day more than individuals from control groups $(95 \%$ confidence interval $[\mathrm{CI}]:[1605,2641]$ steps/day; $p<0.001$ ). Subgroup analyses showed a significantly increased physical activity above baseline in all groups with a chronic disease, including chronic respiratory diseases $(+1314$ steps/day; 95\% CI: [203, 2426] steps/day; $p=0.020)$, T2DM (+2693 steps/day; 95\% CI: [1804, 3581] steps/day; $p<0.001)$, CVD (+ 1300 steps/ day; 95\% CI: $[370,2230]$ steps/day; $p=0.006)$, overweight/obesity (+2405 steps/day; 95\% CI: [1232, 3577] steps/day; $p<0.001)$ and sedentary older adults $(+2568$ steps/day; 95\% CI: [1396, 3740] steps/day; $p<0.001)$, compared to the control groups Fig. 3. However, these results were statistically heterogeneous with respect to the overall effect $\left(Q=375.2, p<0.001 ; \mathrm{I}^{2}=92\right)$ as well as the results from individual subgroups including chronic respiratory diseases $\left(Q=75.9, \mathrm{p}<0.001 ; \mathrm{I}^{2}=97\right)$, T2DM $\left(Q=95.3, \mathrm{p}<0.001 ; \mathrm{I}^{2}=86\right)$, overweight/obesity $(Q=4.4$, $\left.p=0.037 ; \mathrm{I}^{2}=77\right)$ and sedentary older adults $(Q=58.9$, $\left.\mathrm{p}<0.001 ; \mathrm{I}^{2}<82\right)$. In addition, significant publication bias was found in subgroup analyses for patients with 


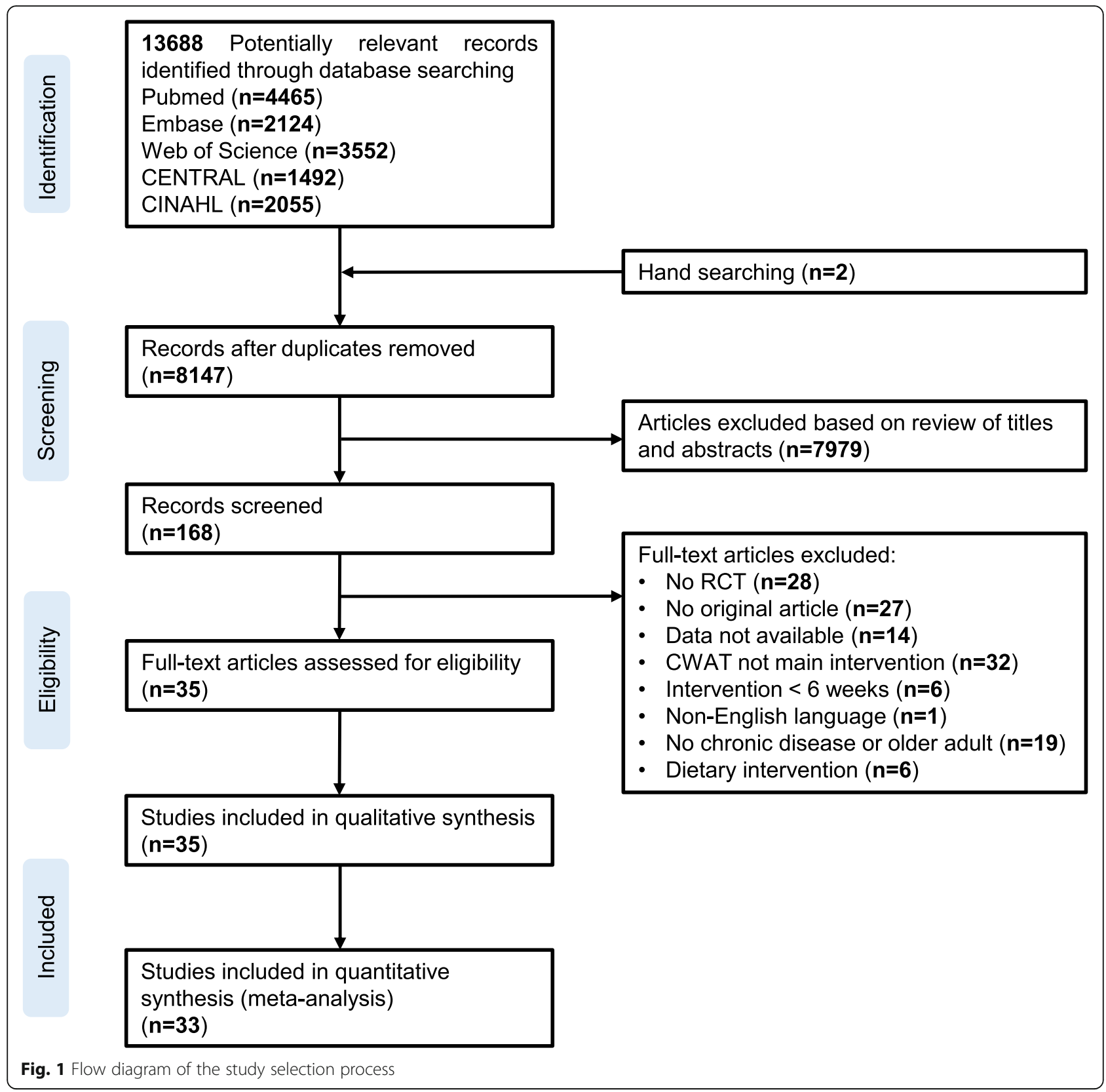

CVD $(p=0.012)$, overweight/obesity $(\mathrm{p}=0.037)$ and sedentary older adults $(p=0.004)$. Since only 1 study was found regarding cognitive diseases, this study was not included into the meta-analysis. No significant improvement in physical activity was found in patients with Alzheimer's disease after an 8-week intervention period.

Association between physical activity and participant and intervention characteristics

Meta-regression analysis showed that all participant characteristics were significantly associated with increased physical activity among individuals from the intervention groups. With respect to the participant characteristics, sex proportion $(\beta=41.0 ; p=0.039)$ and CWAT users of a younger age $(\beta=-189.3 ; p=0.007)$ were significantly associated with a higher increase in physical activity. In addition, patients with chronic respiratory diseases or CVD had a significantly lower increase in physical activity, compared to other populations with chronic diseases $(p<0.001)$. Furthermore, no significant association was found between the intervention duration $(\beta=-7.2 ; p=0.951)$, intervention type $(\beta=-424.4 ; p=0.528)$ and physical activity. 


\section{Random sequence generation (selection bias)}

Allocation concealment (selection bias)

Blinding of participants and personnel (performance bias)

Blinding of outcome assessment (detection bias)

Incomplete outcome data (attrition bias)

Selective reporting (reporting bias)
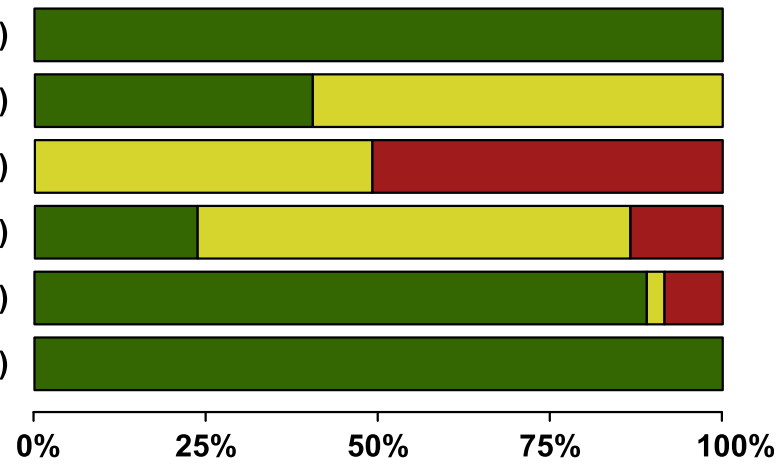

\begin{tabular}{|llll|}
\hline Low risk of bias & $\square$ Unclear risk of bias
\end{tabular} High risk of bias

Fig. 2 Risk of bias graph for included studies $(n=35)$

Physical activity and cardiometabolic health

Participants from the intervention groups significantly decreased their waist circumference $(-0.99 \mathrm{~cm}$; $95 \% \mathrm{CI}$ : $[-1.48,-0.50] \mathrm{cm} ; p<0.001)$, systolic BP $(-3.79 \mathrm{~mm}$ Hg; 95\% CI: [-4.53, - 3.04] mm Hg; $\mathrm{p}<0.001)$ and LDL cholesterol concentration $(-5.70 \mathrm{mg} / \mathrm{dl}$; 95\% CI: [-9.24, - 2.15] $\mathrm{mg} / \mathrm{dl} ; p=0.002)$ more than individuals from the control group (Table 2). In addition, both waist circumference $\left(Q=20.0, p=0.40 ; \mathrm{I}^{2}=12\right)$, systolic $\mathrm{BP}(Q=7.5$, $\left.p=0.98 ; \mathrm{I}^{2}<0.001\right)$ and LDL cholesterol concentration $\left(Q=9.0, p=0.44 ; \mathrm{I}^{2}<0.001\right)$ were all homogeneous results and no significant publication bias was found. Furthermore, the study by Vogel et al. evaluated changes in physical fitness after a 6 -week post-rehabilitation period and showed a significant increased peak workload in the CWAT intervention group (from $185 \pm 55$ to $192 \pm 54 \mathrm{~W}$ [+ $27 \mathrm{~W}] ; p<0.001)$, compared to the control group (from $186 \pm 52$ to $169 \pm 44 \mathrm{~W}[-17 \mathrm{~W}]$ ).

\section{Discussion}

This review systematically evaluated the efficacy of CWAT-based interventions to promote physical activity levels and improve cardiometabolic health in sedentary older adults and patients with chronic diseases, including chronic respiratory diseases, T2DM, CVD, overweight/ obesity and cognitive disorders. To the best of our knowledge, this is the first systematic review and metaanalysis that evaluates the impact of CWATs on physical activity and cardiometabolic health in populations with various chronic diseases. In addition, these results are solely based on randomised controlled trials and objectively measured physical activity (number of steps per day). In general, individuals with a CWAT increased their physical activity level and this may be associated with improvements in cardiometabolic health including waist circumference, systolic BP and LDL cholesterol concentration.
Physical activity levels increased by 2123 steps per day. This is comparable to $20 \mathrm{~min}$ or $1.5 \mathrm{~km}$ of walking. These results are consistent with findings from other meta-analyses showing an increased physical activity among outpatient adults (+ 2500 steps/day) [14] and patients with T2DM (+1822-2042 steps/day) [21, 22]. In addition, de Vries et al. showed that behavioural phyical actvity interventions with an activity monitor increase physical activity (both steps/day and moderate-tovigorous physical activity) in adults with overweight and obesity [20]. Armstrong et al. found that pedometerbased physical activity promotion increased steps per day (+ 1000 steps/day) when it is used as an intervention alone or alongside pulmonary rehabilitation [67]. These studies all confirm that a CWAT-only, or included in a multi-component intervention, have a positive effect in favor of the interventions groups. Here, Dwyer et al. and Jefferis et al. both found an association between allcause mortality and step count where every increment of 1000 steps per day led to a 6 and 14\% risk reduction, respectively $[68,69]$. In addition, the NAVIGATOR study, which consisted of 9000 individuals with high cardiovascular risk or impaired glucose tolerance, has shown that for every additional 2000 steps/day the risk of developing cardiovascular events decreased by $10 \%$, T2DM by $5.5 \%$, and the metabolic syndrome risk score redcued by 0.29 $z$-score [70-72]. In general, CWAT-users included in this meta-analysis increased their step count from 5100 steps per day at baseline to 7200 after the intervention period. Consequently and in accordance with Lee et al., who obeserved that hazard ratios of all-cause mortality declined progressively with higher mean steps per day until approximately 7500 steps/day after which they leveled in older adult women [73], this could indicate a substantial reduction in all-cause mortality risk and the development of chronic diseases. As such, our findings are relevant for the general population and for those 


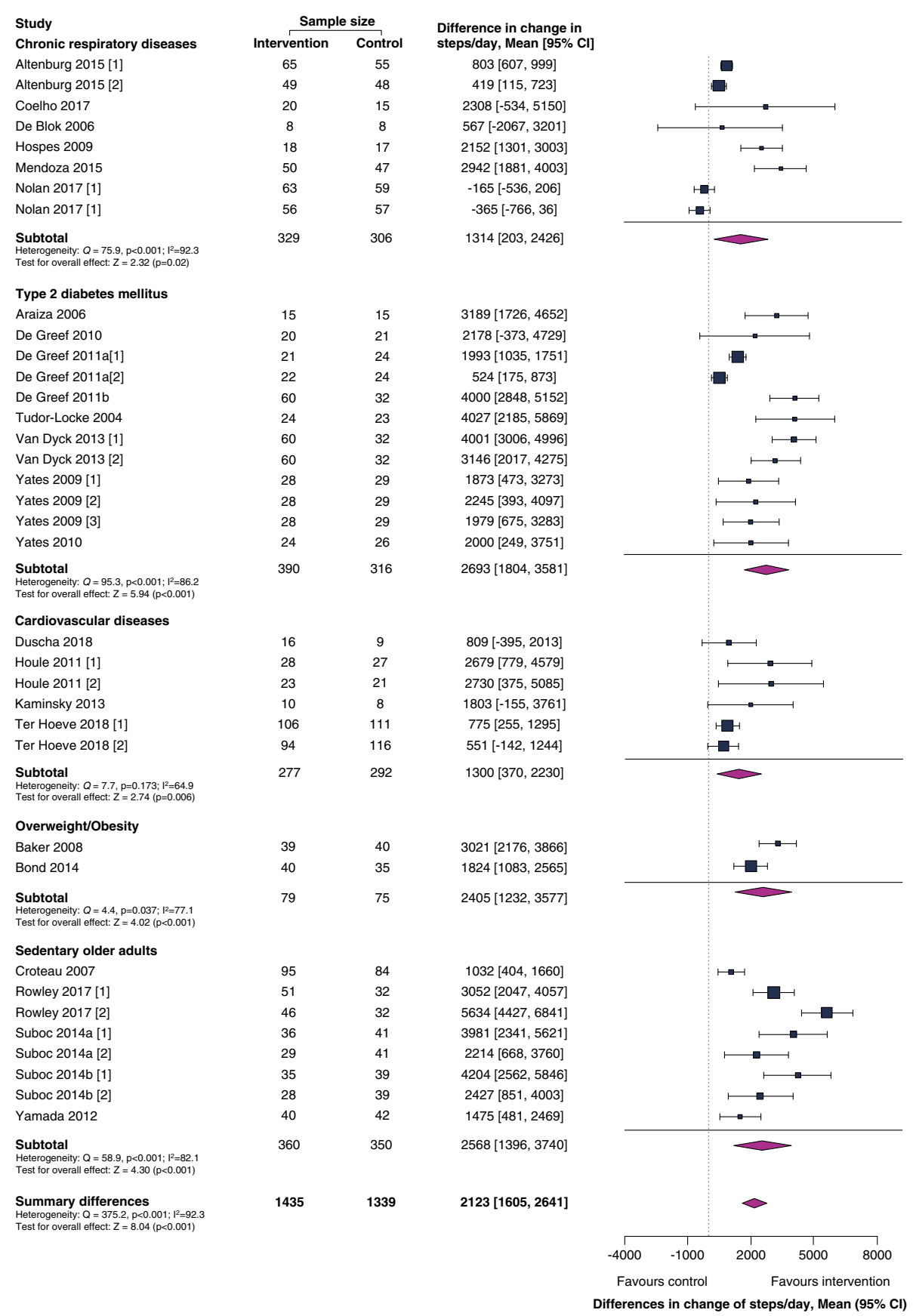

Fig. 3 Forest plot of mean differences of physical activity (steps/day) from CWAT-based behaviour change interventions, compared to control groups. Abbreviations: Cl: confidence interval, $l^{2}$ : the variation in pooled effect size attributable to heterogeneity within that group

who cannot participate in high-intensity physical activities.

Meta-regression analysis showed that both patients with COPD and CVD exhibited the lowest increase in physical activity among all populations with chronic diseases included in this systematic review. This might be due to airflow and cardiac limitations and most of these studies were performed after a rehabilitation period where baseline physical activity levels were reduced. These findings were also found by Amstrong et al., who showed that COPD patients with greater baseline physical activity levels (>4000 steps/day) had greater improvements in steps per day. These results can be confirmed by our results since the baseline physical activity levels of COPD patients in our meta-analysis were 3335 steps per day. In addition, meta-regression analysis 
Table 2 Effects of CWAT-based behaviour change strategies on cardiometabolic health including anthropometrics, cardiovascular health and biochemical parameters

\begin{tabular}{|c|c|c|c|c|c|c|}
\hline \multirow[t]{2}{*}{ Characteristics } & \multirow[t]{2}{*}{ No. of studies } & \multirow[t]{2}{*}{$\begin{array}{l}\text { No. of participants } \\
\text { (intervention/control) }\end{array}$} & \multicolumn{2}{|c|}{$\begin{array}{l}\text { Preintervention mean } \\
\text { (SD) }\end{array}$} & \multirow[t]{2}{*}{$\begin{array}{l}\text { Mean change ( } 95 \% \\
\text { confidence interval) }\end{array}$} & \multirow[t]{2}{*}{ p-value } \\
\hline & & & Intervention & Control & & \\
\hline \multicolumn{7}{|l|}{ Anthropometrics } \\
\hline Body weight (kg) & 11 & $582 / 570$ & $90.6(18.3)$ & $88.4(18.3)$ & $-0.35[-0.84,0.13]$ & 0.15 \\
\hline Body mass index $\left(\mathrm{kg} / \mathrm{m}^{2}\right)$ & 16 & $730 / 733$ & $30.2(4.9)$ & $30.5(5.6)$ & $-0.05[-0.20,0.11]$ & 0.56 \\
\hline Waist circumference (cm) & 13 & $715 / 715$ & $103.6(13.4)$ & $103.3(14.7)$ & $-0.99[-1.48,-0.50]$ & $<0.001$ \\
\hline \multicolumn{7}{|l|}{ Cardiovascular } \\
\hline Systolic blood pressure (mm Hg) & 13 & $655 / 654$ & $133.1(20.0)$ & $131.7(18.2)$ & $-3.79[-4.53,-3.04]$ & $<0.001$ \\
\hline Diastolic blood pressure (mm Hg) & 12 & $535 / 590$ & $77.3(13.9)$ & $77.4(12.0)$ & $0.12[-1.23,1.46]$ & 0.87 \\
\hline Resting heart rate (bpm) & 6 & $295 / 320$ & $68.5(11.6)$ & $67.4(10.5)$ & $-1.92[-3.96,0.13]$ & 0.07 \\
\hline \multicolumn{7}{|l|}{ Biochemical } \\
\hline $\mathrm{HbA1c}(\%)$ & 11 & $462 / 424$ & $7.0(1.4)$ & $7.1(1.4)$ & $-0.07[-0.16,0.01]$ & 0.08 \\
\hline Fasting glucose $(\mathrm{mg} / \mathrm{dl})^{\mathrm{a}}$ & 9 & $471 / 431$ & $124.1(40.1)$ & $116.9(37.3)$ & $-1.67[-5.82,2.49]$ & 0.43 \\
\hline Triglycerides $(\mathrm{mg} / \mathrm{dl})^{\mathrm{a}}$ & 8 & $452 / 414$ & $151.7(96.6)$ & $149.0(103.1)$ & $-1.11[-8.03,5.81]$ & 0.75 \\
\hline Total cholesterol $(\mathrm{mg} / \mathrm{dl})^{\mathrm{a}}$ & 11 & $483 / 459$ & $183.9(44.2)$ & $187.6(49.4)$ & $-1.48[-7.48,4.51]$ & 0.63 \\
\hline HDL cholesterol $(\mathrm{mg} / \mathrm{dl})^{\mathrm{a}}$ & 9 & $484 / 448$ & $46.7(16.1)$ & $48.0(18.0)$ & $0.29[-1.13,1.72]$ & 0.69 \\
\hline LDL cholesterol $(\mathrm{mg} / \mathrm{dll})^{\mathrm{a}}$ & 6 & $408 / 364$ & $98.4(39.8)$ & $102.8(45.3)$ & $-5.70[-9.24,-2.15]$ & 0.002 \\
\hline
\end{tabular}

Abbreviations: LDL: low-density lipoprotein, HDL: High-density lipoprotein

${ }^{a}$ Blood parameters were converted to the same unit (from $\mathrm{mmol} / \mathrm{l}$ to $\mathrm{mg} / \mathrm{dl}$ ), including triglycerides (divide by 0.0112 ), total cholesterol, $\mathrm{HDL}$ cholesterol, $\mathrm{LDL}$ cholesterol (divide by 0.02586 ) and glucose (divide by 0.05551 ) concentration

showed no additional effect of behaviour change strategies (goal setting, group counselling, individual counselling or telephone support) on physical activity levels. This is in contrast with a systematic review that showed a positive effect for CWATs, even when interventions were separated into CWAT-only and multifaceted interventions [74]. However, a larger effect size for interventions that were multifaceted was found compared to CWAT-only interventions. This suggests that CWATs can be effective on their own, but when combined with other behaviour change strategies, such as telephone support or group-based counselling, the improvement in physical activity is greater. However, it must be outlined that Brickwood et al. only included healthy adults and there was only a small number of studies in our meta-regression that included both a CWAT-only and CWATs as part of a multi-component intervention group. Therefore, more studies are warranted which include both a CWAT-only and a multi-component intervention group. Furthermore, patient characteristics were all independently associated with an increased number of step, which possibly explains the heterogeneity of the overall effect and subgroup analyses. For example, the obese population was younger of age compared to other populations and, as a result, propably showed a higher increase in physical activity. Therefore, caution is warranted when interpreting these results.
The increment of physical activity was associated with improvements in several cardiometabolic health parameters. The systolic BP was significantly decreased in the intervention groups. This finding is consistent with previous systematic reviews with respect to physical activity and blood pressure among outpatient [14] and healthy sedentary adults $[75,76]$. It has been shown that a change of $-10 \mathrm{~mm} \mathrm{Hg}$ in systolic BP reduces the risk of developing CVD by $17 \%$ and overall mortality by $13 \%$ [77]. In addition, a decrease in systolic BP of $2 \mathrm{~mm} \mathrm{Hg}$ is associated with a reduction of $10 \%$ in stroke mortality and a $7 \%$ reduction in mortality originating from CVD [78]. Furthermore, a significantly reduced LDL cholsterol concentration was found when physical activity levels were increased among individuals with CWATs. A meta-analysis from The Cholesterol Treatment Trialists' Collaboration showed that with the aid of statins the 5year incidence of major vascular events reduced by about $20 \%$ and all-cause mortality by $12 \%$ per $40 \mathrm{mg} / \mathrm{dl}$ in LDL cholesterol concentration. However, since we showed a reduction of $6 \mathrm{mg} / \mathrm{dl}$, medication has probably more beneficial effects rather than increasing physical activity [79]. In addition, most data regarding physical activity and serum lipids seem to indicate that regular physical activity or exercise training does not reduce LDL cholesterol concentration in a clinically relevant way [80, 81]. However, it has been suggested that regular physical activity may change the LDL cholesterol particle 
size, even when total LDL concentrations remain constant $[82,83]$. We also found a significantly decreased waist circumference with the same magnitude $(-1.19$ $\mathrm{cm}$; 95\% CI: [-1.79, -0.59$] \mathrm{cm}$ ) as found in another published meta-analysis in which the effect of physical activity and dietery interventions on waist circumference was evaluated [11]. De Koning et al. have shown that a decrease of $1 \mathrm{~cm}$ in waist circumference reduces the relative risk of a CVD event by $2 \%$ [84]. In this way, CWAT devices can improve health related outcomes which are closely related to CVD.

Several limitations of the included studies were observed. First, studies with relatively small sample sizes and different intervention durations were included in the meta-analyses. In addition, this review reflected heterogeneous study designs which consisted of multiple components including CWAT use, goal setting, telephone calls, diary use, individual or group counselling, different therapists, face-to-face or written motivation and the frequency of the sessions. Therefore, the independent contribution of any of these components, or a combination of these factors, is difficult to establish. This probably explains the high heterogeneity of the physical activity results $\left(\mathrm{Q}=375, p<0.001 ; \mathrm{I}^{2}=92\right)$. However, since clinical and methodological diversity is always present in meta-analysis statistical heterogeneity is inevitable. Moreover, Higgens et al. reported that almost one third of meta-analyses have moderate to considerable heterogeneity [85]. Secondly, only eleven studies evaluated physical activty (steps/day) in combination with one or more of the cardiometabolic risk factors. In this way, no direct associations between increased physical acitivities (steps/day) and cardiometabolic risk factors could be made and no minimal amount of steps per day to improve cardiometabolic health could be determined. Thirdly, various CWAT devices were both used to objectively measure physical activity and as a motivational instrument. As a result, this may affect the control group by increasing their physical activity by the thought that they were being monitored. Finally, all studies only measured physical activity but did not take into account sedentary time. It has been shown that sedentary behaviour is a risk factor for cardiometabilic health and all-cause mortality, indepently of the amount of physical activity [86, 87]. Therefore, we recommend to include sedentary time, next to physical activity, in future studies.

\section{Conclusion}

These results suggest that populations with chronic diseases significantly increase physical activity using CWATs only, or as part of a multi-component intervention, and improve their cardiometabolic health such as a reduced waist circumference, systolic BP and LDL cholesterol concentration.

\section{Supplementary information}

Supplementary information accompanies this paper at https://doi.org/10. 1186/s12966-020-00955-2.

Additional file 1: Appendix I Pubmed search.

Additional file 2: Appendix II Data extraction form.

Additional file 3: Appendix III Funnel plots.

\begin{abstract}
Abbreviations
CWAT: Consumer wearable activity tracker; T2DM: Type 2 diabetes mellitus; CVD: Cardiovascular diseases; WHO: World Health Organization;

PRISMA: Preferred Reporting Items for Systematic reviews and Meta-Analysis; CENTRAL: Cochrane Central Register of Controlled Trials; BMI: Body Mass Index; BP: Blood pressure; HDL: High-density lipoprotein; LDL: Low-density lipoprotein; HbA1c: Blood glycated haemoglobin concentration; COPD: Chronic obstructive pulmonary disease
\end{abstract}

\section{Acknowledgements}

Not applicable.

\section{Authors' contributors}

W.M.A.F and B.O.E contributed to the design of the study, generated hypotheses, extracted and interpreted the data and wrote and critically reviewed the manuscript. G.H.L.M.F contributed to the development of the search strategy and wrote and critically reviewed the manuscript. J. S extracted and interpreted the data and critically reviewed the manuscript. F.S. performed the statitical analyses, interpreted the data and critically reviewed the manuscript.

\section{Funding}

The authors have not declared a specific grant for this research from any funding agency in the public, commercial or not-for-profit sectors.

\section{Availability of data and materials}

All data generated or analysed during this systematic review are included in this published article [and its supplementary information files].

\section{Ethics approval and consent to participate}

Not applicable.

\section{Consent for publication}

Not applicable.

\section{Competing interests}

The authors declare that they have no competing interests.

\section{Author details}

${ }^{1}$ REVAL - Rehabilitation Research Center, Faculty of Rehabilitation Sciences, Hasselt University, Hasselt, Belgium. ${ }^{2}$ BIOMED - Biomedical Research Institute, Faculty of Medicine and Life Sciences, Hasselt University, Hasselt, Belgium. ${ }^{3}$ Department of Education and Research Support, University Library, Maastricht University, Maastricht, The Netherlands. ${ }^{4}$ Data Science Institute for Biostatistics and Statistical Bioinformatics, Hasselt University, Hasselt, Belgium. ${ }^{5}$ ADLON Sports Medical Center, Hasselt, Belgium.

Received: 19 December 2019 Accepted: 6 April 2020

Published online: 11 May 2020

\section{References}

1. Organization WH. Global action plan for the prevention and control of noncommunicable diseases 2013-2020. Geneva; 2013.

2. Vos T, Barber RM, Bell B, Bertozzi-Villa A, Biryukov S, Bolliger I, et al. Global, regional, and national incidence, prevalence, and years lived with disability for 301 acute and chronic diseases and injuries in 188 countries, 1990-2013: 
a systematic analysis for the global burden of disease study 2013. Lancet. 2015;386(9995):743-800.

3. WHO. Global action plan for the prevention and control of noncommunicable diseases. 2013-2020:2013.

4. Lee I-M, Shiroma EJ, Lobelo F, Puska P, Blair SN, Katzmarzyk PT, et al. Effect of physical inactivity on major non-communicable diseases worldwide: an analysis of burden of disease and life expectancy. Lancet. 2012;380(9838): 219-29.

5. Garcia-Aymerich J, Lange P, Benet M, Schnohr P, Antó JM. Regular physical activity reduces hospital admission and mortality in chronic obstructive pulmonary disease: a population based cohort study. Thorax. 2006;61(9): $772-8$.

6. Pedersen BK, Saltin B. Exercise as medicine-evidence for prescribing exercise as therapy in 26 different chronic diseases. Scand J Med Sci Sports. 2015;25(S3):1-72.

7. organization WH. Global recommendations on physical activity for health. Geneva2010.

8. Morgan PJ, Young MD, Smith JJ, Lubans DR. Targeted health behavior interventions promoting physical activity: a conceptual model. Exerc Sport Sci Rev. 2016;44(2):71-80.

9. WHO. Global action plan on physical activity 2018-2030: more active people for a healthier world. More active people for a healthier world. Geneva: World Health Organization. 2018.

10. Bock C, Jarczok MN, Litaker D. Community-based efforts to promote physical activity: a systematic review of interventions considering mode of delivery, study quality and population subgroups. J Sci Med Sport. 2014; 17(3):276-82.

11. Patnode CD, Evans CV, Senger CA, Redmond N, Lin JS. Behavioral counseling to promote a healthful diet and physical activity for cardiovascular disease prevention in adults without known cardiovascular disease risk factors: updated evidence report and systematic review for the US preventive services task force. Jama. 2017;318(2):175-93.

12. Cradock KA, G OL, Finucane FM, Gainforth HL, Quinlan LR, Ginis KA. Behaviour change techniques targeting both diet and physical activity in type 2 diabetes: A systematic review and meta-analysis. The international journal of behavioral nutrition and physical activity. 2017;14(1):18.

13. Goode AD, Reeves MM, Eakin EG. Telephone-delivered interventions for physical activity and dietary behavior change: an updated systematic review. Am J Prev Med. 2012;42(1):81-8.

14. Bravata DM, Smith-Spangler C, Sundaram V, Gienger AL, Lin N, Lewis R, et al. Using pedometers to increase physical activity and improve health: a systematic review. Jama. 2007;298(19):2296-304.

15. Coughlin SS, Stewart J. Use of Consumer Wearable Devices to Promote Physical Activity: A Review of Health Intervention Studies. Journal of environment and health sciences. 2016;2(6).

16. An H-S, Jones GC, Kang S-K, Welk GJ, Lee J-M. How valid are wearable physical activity trackers for measuring steps? Eur J Sport Sci. 2017;17(3):360-8.

17. Evenson KR, Goto MM, Furberg RD. Systematic review of the validity and reliability of consumer-wearable activity trackers. Int I Behav Nutr Phys Act. 2015;12(1):159

18. Kooiman TJ, Dontje ML, Sprenger SR, Krijnen WP, van der Schans CP, de Groot M. Reliability and validity of ten consumer activity trackers. BMC sports science, medicine and rehabilitation. 2015;7(1):24.

19. Baskerville R, Ricci-Cabello I, Roberts N, Farmer A. Impact of accelerometer and pedometer use on physical activity and glycaemic control in people with type 2 diabetes: a systematic review and meta-analysis. Diabetic medicine : a journal of the British Diabetic Association. 2017;34(5):612-20.

20. de Vries HJ, Kooiman TJ, van Ittersum MW, van Brussel M, de Groot M. Do activity monitors increase physical activity in adults with overweight or obesity? A systematic review and meta-analysis. Obesity (Silver Spring). 2016;24(10):2078-91.

21. Qiu S, Cai X, Chen X, Yang B, Sun Z. Step counter use in type 2 diabetes: a meta-analysis of randomized controlled trials. BMC Med. 2014;12:36.

22. Vaes AW, Cheung A, Atakhorrami M, Groenen MT, Amft O, Franssen FM, et al. Effect of 'activity monitor-based' counseling on physical activity and health-related outcomes in patients with chronic diseases: a systematic review and meta-analysis. Ann Med. 2013:45(5-6):397-412.

23. Qiu SH, Cai X, Wang XY, He C, Zugel M, Steinacker JM, et al. Using step counters to promote physical activity and exercise capacity in patients with chronic obstructive pulmonary disease: a meta-analysis. Ther Adv Respir Dis. 2018;12:14.
24. Moher D, Liberati A, Tetzlaff J, Altman DG, Group P. Preferred reporting items for systematic reviews and meta-analyses: the PRISMA statement. PLoS Med. 2009;6(7):e1000097.

25. Liberati A, Altman DG, Tetzlaff J, Mulrow C, Gøtzsche PC, loannidis JP, et al. The PRISMA statement for reporting systematic reviews and meta-analyses of studies that evaluate health care interventions: explanation and elaboration. PLoS Med. 2009;6(7):e1000100.

26. Bramer WM, Giustini D, de Jonge GB, Holland L, Bekhuis T. De-duplication of database search results for systematic reviews in EndNote. Journal of the Medical Library Association: JMLA. 2016;104(3):240.

27. Higgins JP, Green S. Cochrane handbook for systematic reviews of interventions. Version 5.2; 2017.

28. Hozo SP, Djulbegovic B, Hozo I. Estimating the mean and variance from the median, range, and the size of a sample. BMC Med Res Methodol. 2005;5:13.

29. Laposata M. Laboratory Medicine: The Diagnosis of Disease in the Clinical Laboratory. 2nd ed2014.

30. Team RC. R: a language and environment for statistical computing; 2013.

31. Higgins JP, Thompson SG, Deeks JJ, Altman DG. Measuring inconsistency in meta-analyses. BMJ: British Medical Journal. 2003:327(7414):557.

32. Altenburg WA, ten Hacken NH, Bossenbroek L, Kerstjens HA, de Greef $M H$, Wempe JB. Short- and long-term effects of a physical activity counselling programme in COPD: a randomized controlled trial. Respir Med. 2015;109(1): $112-21$.

33. Coelho CM, Reboredo MM, Valle FM, Malaguti C, Campos LA, Nascimento $L M$, et al. Effects of an unsupervised pedometer-based physical activity program on daily steps of adults with moderate to severe asthma: a randomized controlled trial. J Sports Sci. 2018;36(10):1186-93.

34. de Blok BM, de Greef MH, ten Hacken NH, Sprenger SR, Postema K, Wempe JB. The effects of a lifestyle physical activity counseling program with feedback of a pedometer during pulmonary rehabilitation in patients with COPD: a pilot study. Patient Educ Couns. 2006;61(1):48-55.

35. Hospes G, Bossenbroek L, Ten Hacken NH, van Hengel P, de Greef MH. Enhancement of daily physical activity increases physical fitness of outclinic COPD patients: results of an exercise counseling program. Patient Educ Couns. 2009;75(2):274-8.

36. Kawagoshi A, Kiyokawa N, Sugawara K, Takahashi H, Sakata S, Satake M, et al. Effects of low-intensity exercise and home-based pulmonary rehabilitation with pedometer feedback on physical activity in elderly patients with chronic obstructive pulmonary disease. Respir Med. 2015; 109(3):364-71.

37. Mendoza L, Horta P, Espinoza J, Aguilera M, Balmaceda N, Castro A, et al. Pedometers to enhance physical activity in COPD: a randomised controlled trial. Eur Respir J. 2015;45(2):347-54.

38. Nolan CM, Maddocks M, Canavan JL, Jones SE, Delogu V, Kaliaraju D, et al. Pedometer step count targets during pulmonary rehabilitation in chronic obstructive pulmonary disease. A randomized controlled trial. Am J Respir Crit Care Med. 2017:195(10):1344-52.

39. Araiza P, Hewes H, Gashetewa C, Vella CA, Burge MR. Efficacy of a pedometer-based physical activity program on parameters of diabetes control in type 2 diabetes mellitus. Metab Clin Exp. 2006;55(10):1382-7.

40. Bjorgaas MR, Vik JT, Stolen T, Lydersen S, Grill V. Regular use of pedometer does not enhance beneficial outcomes in a physical activity intervention study in type 2 diabetes mellitus. Metab Clin Exp. 2008;57(5):605-11.

41. De Greef K, Deforche B, Tudor-Locke C, De Bourdeaudhuij I. A cognitivebehavioural pedometer-based group intervention on physical activity and sedentary behaviour in individuals with type 2 diabetes. Health Educ Res. 2010;25(5):724-36.

42. De Greef K, Deforche B, Tudor-Locke C, De Bourdeaudhuij I. Increasing physical activity in Belgian type 2 diabetes patients: a three-arm randomized controlled trial. International journal of behavioral medicine. 2011;18(3):188-98.

43. De Greef KP, Deforche BI, Ruige JB, Bouckaert JJ, Tudor-Locke CE, Kaufman $J M$, et al. The effects of a pedometer-based behavioral modification program with telephone support on physical activity and sedentary behavior in type 2 diabetes patients. Patient Educ Couns. 2011;84(2):275-9.

44. Diedrich A, Munroe DJ, Romano M. Promoting physical activity for persons with diabetes. The Diabetes educator. 2010;36(1):132-40.

45. Engel $\mathrm{L}$, Lindner $\mathrm{H}$. Impact of using a pedometer on time spent walking in older adults with type 2 diabetes. The Diabetes educator. 2006;32(1):98-107.

46. Kooiman TJM, de Groot M, Hoogenberg K, Krijnen WP, van der Schans CP, Kooy A. Self-tracking of physical activity in people with type 2 diabetes: a 
randomized controlled trial. Computers, informatics, nursing : CIN. 2018; 36(7):340-9.

47. Tudor-Locke C, Bell RC, Myers AM, Harris SB, Ecclestone NA, Lauzon N, et al. Controlled outcome evaluation of the first step program: a daily physical activity intervention for individuals with type II diabetes. International journal of obesity and related metabolic disorders : journal of the International Association for the Study of Obesity. 2004;28(1):113-9.

48. Van Dyck D, De Greef K, Deforche B, Ruige J, Bouckaert J, Tudor-Locke CE, et al. The relationship between changes in steps/day and health outcomes after a pedometer-based physical activity intervention with telephone support in type 2 diabetes patients. Health Educ Res. 2013;28(3):539-45.

49. Yates T, Davies M, Gorely T, Bull F, Khunti K. Effectiveness of a pragmatic education program designed to promote walking activity in individuals with impaired glucose tolerance: a randomized controlled trial. Diabetes Care. 2009;32(8):1404-10.

50. Yates T, Davies MJ, Gorely T, Talbot D, Bull F, Sattar N, et al. The effect of increased ambulatory activity on markers of chronic low-grade inflammation: evidence from the PREPARE programme randomized controlled trial. Diabetic medicine : a journal of the British Diabetic Association. 2010;27(11):1256-63.

51. Butler L, Furber S, Phongsavan P, Mark A, Bauman A. Effects of a pedometer-based intervention on physical activity levels after cardiac rehabilitation: a randomized controlled trial. Journal of cardiopulmonary rehabilitation and prevention. 2009;29(2):105-14.

52. Duscha BD, Piner LW, Patel MP, Craig KP, Brady M, McGarrah RW 3rd, et al. Effects of a 12-week mHealth program on peak VO2 and physical activity patterns after completing cardiac rehabilitation: a randomized controlled trial. Am Heart J. 2018;199:105-14.

53. Houle J, Doyon O, Vadeboncoeur N, Turbide G, Diaz A, Poirier P. Innovative program to increase physical activity following an acute coronary syndrome: randomized controlled trial. Patient Educ Couns. 2011;85(3): e237-44.

54. Kaminsky LA, Jones J, Riggin K, Strath SJ. A pedometer-based physical activity intervention for patients entering a maintenance cardiac rehabilitation program: a pilot study. Cardiovascular diagnosis and therapy. 2013;3(2):73-9

55. Ter Hoeve N, Sunamura M, Stam HJ, Boersma E, Geleijnse ML, van Domburg RT, et al. Effects of two behavioral cardiac rehabilitation interventions on physical activity: a randomized controlled trial. Int J Cardiol. 2018;255:221-8.

56. Vogel J, Auinger A, Riedl R, Kindermann H, Helfert M, Ocenasek H. Digitally enhanced recovery: investigating the use of digital self-tracking for monitoring leisure time physical activity of cardiovascular disease (CVD) patients undergoing cardiac rehabilitation. PLoS One. 2017;12(10):e0186261.

57. Baker G, Gray SR, Wright A, Fitzsimons C, Nimmo M, Lowry R, et al. The effect of a pedometer-based community walking intervention "walking for wellbeing in the west" on physical activity levels and health outcomes: a 12-week randomized controlled trial. The international journal of behavioral nutrition and physical activity. 2008:5:44.

58. Bond DS, Vithiananthan S, Thomas JG, Trautvetter J, Unick JL, Jakicic JM, et al. Bari-active: a randomized controlled trial of a preoperative intervention to increase physical activity in bariatric surgery patients. Surgery for obesity and related diseases : official journal of the American Society for Bariatric Surgery. 2015;11(1):169-77.

59. Kempf K, Rohling M, Stichert M, Fischer G, Boschem E, Konner J, et al. Telemedical coaching improves long-term weight loss in overweight persons: a randomized controlled trial. Int J Telemed Appl. 2018;2018:7530602.

60. Vidoni ED, Watts AS, Burns JM, Greer CS, Graves RS, Van Sciver A, et al. Feasibility of a memory clinic-based physical activity prescription program. Journal of Alzheimer's disease : JAD. 2016;53(1):161-70.

61. Armit CM, Brown WJ, Marshall AL, Ritchie CB, Trost SG, Green A, et al. Randomized trial of three strategies to promote physical activity in general practice. Prev Med. 2009;48(2):156-63.

62. Croteau KA, Richeson NE, Farmer BC, Jones DB. Effect of a pedometer-based intervention on daily step counts of community-dwelling older adults. Res Q Exerc Sport. 2007;78(5):401-6.

63. Rowley TW, Lenz EK, Swartz AM, Miller NE, Maeda H, Strath SJ. Efficacy of an individually tailored, internet-mediated physical activity intervention in older adults: a randomized controlled trial. Journal of applied gerontology : the official journal of the Southern Gerontological Society. 2019;38(7):1011-22.

64. Suboc TB, Strath SJ, Dharmashankar K, Harmann L, Couillard A, Malik M, et al. The impact of moderate intensity physical activity on cardiac structure and performance in older sedentary adults. International journal of cardiology Heart \& vessels. 2014;4:19-24.

65. Yamada M, Mori S, Nishiguchi S, Kajiwara Y, Yoshimura K, Sonoda T, et al. Pedometer-based behavioral change program can improve dependency in sedentary older adults: a randomized controlled trial. The Journal of frailty \& aging. 2012;1 (1):39-44.

66. Suboc TB, Strath SJ, Dharmashankar K, Coulliard A, Miller N, Wang J, et al. Relative importance of step count, intensity, and duration on physical activity's impact on vascular structure and function in previously sedentary older adults. J Am Heart Assoc. 2014;3(1):e000702.

67. Armstrong M, Winnard A, Chynkiamis N, Boyle S, Burtin C, Vogiatzis I. Use of pedometers as a tool to promote daily physical activity levels in patients with COPD: a systematic review and meta-analysis. European respiratory review : an official journal of the European Respiratory Society. 2019;28(154).

68. Dwyer T, Pezic A, Sun C, Cochrane J, Venn A, Srikanth V, et al. Objectively measured daily steps and subsequent long term all-cause mortality: the Tasped prospective cohort study. PLoS One. 2015;10(11):e0141274.

69. Jefferis BJ, Parsons TJ, Sartini C, Ash S, Lennon LT, Papacosta O, et al. Objectively measured physical activity, sedentary behaviour and all-cause mortality in older men: does volume of activity matter more than pattern of accumulation? Br J Sports Med. 2019;53(16):1013-20.

70. Yates T, Haffner SM, Schulte PJ, Thomas L, Huffman KM, Bales CW, et al. Association between change in daily ambulatory activity and cardiovascular events in people with impaired glucose tolerance (NAVIGATOR trial): a cohort analysis. Lancet (London, England). 2014;383(9922):1059-66.

71. Kraus WE, Yates T, Tuomilehto J, Sun JL, Thomas L, McMurray JJV, et al. Relationship between baseline physical activity assessed by pedometer count and new-onset diabetes in the NAVIGATOR trial. BMJ Open Diabetes Res Care. 2018;6(1):e000523.

72. Huffman KM, Sun JL, Thomas L, Bales CW, Califf RM, Yates T, et al. Impact of baseline physical activity and diet behavior on metabolic syndrome in a pharmaceutical trial: results from NAVIGATOR. Metab Clin Exp. 2014;63(4): 554-61.

73. Lee IM, Shiroma EJ, Kamada M. Bassett DR. Buring JE. Association of Step Volume and Intensity With All-Cause Mortality in Older Women. JAMA internal medicine: Matthews CE; 2019.

74. Brickwood K, Watson G, O'Brien J, Williams AD. Consumer-based wearable activity trackers increase physical activity participation: systematic review and meta-analysis. JMIR mHealth uHealth. 2019;7(4):e11819.

75. Murphy MH, Nevill AM, Murtagh EM, Holder RL. The effect of walking on fitness, fatness and resting blood pressure: a meta-analysis of randomised, controlled trials. Prev Med. 2007;44(5):377-85.

76. Kelley GA, Kelley KS, Tran ZV. Walking and resting blood pressure in adults: a meta-analysis. Prev Med. 2001;33(2 Pt 1):120-7.

77. Ettehad D, Emdin CA, Kiran A, Anderson SG, Callender T, Emberson J, et al. Blood pressure lowering for prevention of cardiovascular disease and death: a systematic review and meta-analysis. Lancet (London, England). 2016; 387(10022):957-67.

78. Lewington S, Clarke R, Qizilbash N, Peto R, Collins R. Age-specific relevance of usual blood pressure to vascular mortality: a meta-analysis of individual data for one million adults in 61 prospective studies. Lancet (London, England). 2002;360(9349):1903-13.

79. Baigent C, Keech A, Kearney PM, Blackwell L, Buck G, Pollicino C, et al. Efficacy and safety of cholesterol-lowering treatment: prospective metaanalysis of data from 90,056 participants in 14 randomised trials of statins. Lancet (London, England). 2005;366(9493):1267-78.

80. Yu CM, Li LS, Ho HH, Lau CP. Long-term changes in exercise capacity, quality of life, body anthropometry, and lipid profiles after a cardiac rehabilitation program in obese patients with coronary heart disease. Am J Cardiol. 2003;91(3):321-5.

81. Wosornu D, Bedford D, Ballantyne D. A comparison of the effects of strength and aerobic exercise training on exercise capacity and lipids after coronary artery bypass surgery. Eur Heart J. 1996;17(6):854-63.

82. Halle M, Berg A, Konig D, Keul J, Baumstark MW. Differences in the concentration and composition of low-density lipoprotein subfraction particles between sedentary and trained hypercholesterolemic men. Metab Clin Exp. 1997:46(2):186-91.

83. Williams PT, Stefanick ML, Vranizan KM, Wood PD. The effects of weight loss by exercise or by dieting on plasma high-density lipoprotein (HDL) levels in men with low, intermediate, and normal-to-high HDL at baseline. Metab Clin Exp. 1994;43(7):917-24. 
84. de Koning L, Merchant AT, Pogue J, Anand SS. Waist circumference and waist-to-hip ratio as predictors of cardiovascular events: meta-regression analysis of prospective studies. Eur Heart J. 2007;28(7):850-6.

85. Higgins JP, Thompson SG, Deeks JJ, Altman DG. Measuring inconsistency in meta-analyses. BMJ (Clinical research ed). 2003;327(7414):557-60.

86. Chau JY, Grunseit AC, Chey T, Stamatakis E, Brown WJ, Matthews CE, et al. Daily sitting time and all-cause mortality: a meta-analysis. PLoS One. 2013; 8(11):e80000.

87. van der Berg JD, Stehouwer CD, Bosma H, van der Velde JH, Willems PJ, Savelberg $\mathrm{HH}$, et al. Associations of total amount and patterns of sedentary behaviour with type 2 diabetes and the metabolic syndrome: the Maastricht study. Diabetologia. 2016;59(4):709-18.

\section{Publisher's Note}

Springer Nature remains neutral with regard to jurisdictional claims in published maps and institutional affiliations.

Ready to submit your research? Choose BMC and benefit from:

- fast, convenient online submission

- thorough peer review by experienced researchers in your field

- rapid publication on acceptance

- support for research data, including large and complex data types

- gold Open Access which fosters wider collaboration and increased citations

- maximum visibility for your research: over $100 \mathrm{M}$ website views per year

At $B M C$, research is always in progress.

Learn more biomedcentral.com/submissions 